\title{
Conductance of Sidewall-Functionalized Carbon Nanotubes: Universal Dependence on Adsorption Sites
}

\author{
García-Lastra, J.M.; Thygesen, Kristian Sommer; Strange, Mikkel; Rubio, Á.
}

Published in:

Physical Review Letters

Link to article, DOI:

10.1103/PhysRevLett.101.236806

Publication date:

2008

Document Version

Publisher's PDF, also known as Version of record

Link back to DTU Orbit

Citation (APA):

García-Lastra, J. M., Thygesen, K. S., Strange, M., \& Rubio, Á. (2008). Conductance of Sidewall-Functionalized Carbon Nanotubes: Universal Dependence on Adsorption Sites. Physical Review Letters, 101(23), 236806. https://doi.org/10.1103/PhysRevLett.101.236806

\section{General rights}

Copyright and moral rights for the publications made accessible in the public portal are retained by the authors and/or other copyright owners and it is a condition of accessing publications that users recognise and abide by the legal requirements associated with these rights.

- Users may download and print one copy of any publication from the public portal for the purpose of private study or research.

- You may not further distribute the material or use it for any profit-making activity or commercial gain

- You may freely distribute the URL identifying the publication in the public portal 


\title{
Conductance of Sidewall-Functionalized Carbon Nanotubes: Universal Dependence on Adsorption Sites
}

\author{
Juan María García-Lastra, ${ }^{1}$ Kristian S. Thygesen, ${ }^{2}$ Mikkel Strange, ${ }^{2}$ and Ángel Rubio ${ }^{1}$ \\ ${ }^{1}$ Nano-Bio Spectroscopy Group and European Theoretical Spectroscopy Facility (ETSF), Departamento de Fisica de Materiales, \\ Unidad de Materiales Centro Mixto CSIC-UPV/EHU, Universidad del Pais Vasco, Avd. Tolosa 72, E-20018 Donostia, Spain \\ ${ }^{2}$ Center for Atomic-scale Materials Design (CAMD), Department of Physics, Technical University of Denmark, \\ DK-2800 Kgs. Lyngby, Denmark
}

(Received 22 April 2008; published 5 December 2008)

\begin{abstract}
We use density functional theory to study the effect of molecular adsorbates on the conductance of metallic carbon nanotubes (CNT). The five molecules considered $\left(\mathrm{NO}_{2}, \mathrm{NH}_{2}, \mathrm{H}, \mathrm{COOH}, \mathrm{OH}\right)$ lead to very similar scattering of the electrons. The adsorption of a single molecule suppresses one of the two available transport channels at the Fermi level while the other is left undisturbed. If more molecules are adsorbed on the same sublattice, the remaining open channel may or may not be blocked, depending on the relative position of the adsorbates. If the relative positions satisfy a simple geometric condition, this channel remains fully open independently of the number of adsorbed molecules.
\end{abstract}

DOI: 10.1103/PhysRevLett.101.236806

Sidewall chemical functionalization of carbon nanotubes (CNTs) has been the subject of several studies (see reviews in [1-3]) as it represents a very direct way to implement a number of technological applications including nanoelectronics devices and various types of sensors [3]. These applications all build on the idea that the electrical conductivity of the nanotube can be controlled externally by adding/removing different functionalized groups [4].

Much theoretical work has been devoted to the analysis of the impact of lattice symmetry and potential impurity or defect scattering in the $I / V$ characteristics of CNTs $[1,3,5-7]$, not only in single wall CNTs, but also in double-wall CNTs [8]. Those studies indicate that the number of available conducting channels in metallic nanotubes depends on the range of the scattering and the chirality of the tube. However, very little is known about the impact of bipartite-lattice symmetry of graphene when multiple scattering centers are present.

In this Letter we show that the transport properties of functionalized CNTs in the phase-coherent regime show a peculiar, systematic dependence on the positions of the adsorbed molecules. Namely, for any number of molecules adsorbed on the same sublattice, the conductivity of the tube remains close to $1 G_{0}$ if the relative positions of the adsorbates satisfy a simple geometric condition, whereas the conductance is strongly suppressed if one or more molecules fail to satisfy the condition. As a second result, we show that the change in conductivity of a metallic CNT due to chemisorption of a molecule at an on-top site of the nanotube sidewall is largely independent of the molecular specie. This universal behavior is verified for $\mathrm{H}, \mathrm{COOH}$, $\mathrm{OH}, \mathrm{NH}_{2}$, and $\mathrm{NO}_{2}$ adsorbed on $(6,6)$ armchair, $(9,0)$ zigzag, and $(8,2)$ chiral CNTs.

Recall that a graphene sheet contains two inequivalent types of sites, $A$ and $B$, constituting the two sublattices of graphene. The $p_{z}$ orbitals of the carbon atoms form the $\pi-$
PACS numbers: 73.63.Fg, 31.15.B-, 71.15.Ap, 73.22.-f

and $\pi^{*}-$ valence and conduction bands which intersect at the $K$ and $K^{\prime}$ points of the Brillouin zone (BZ). When a graphene sheet is rolled up to form a CNT, the band structure (neglecting curvature effects) is obtained by restricting the band structure of graphene to discrete lines in the BZ. Metallic CNTs thus have two available channels for transporting electrons (in both directions along the tube axis) at energies close to the Fermi level formed by the Bloch states in the vicinity of the $K$ and $K^{\prime}$ points. We shall refer to these states as forward- and backward-moving states and denote them by $\left|K_{+}\right\rangle,\left|K_{+}^{\prime}\right\rangle$ and $\left|K_{-}\right\rangle,\left|K_{-}^{\prime}\right\rangle$, respectively.

Within a tight-binding (TB) model we can now address how the nanotube electronic response is perturbed by the presence of one molecule adsorbed on the CNT sidewall. To this end we consider the TB model of the CNT with a single level of energy $\varepsilon_{0}$ coupled to one of the $p_{z}$ orbitals of the CNT via the hopping parameter $t$. We can take $\varepsilon_{0}$ to be negative or positive corresponding to a model for the highest occupied molecular orbital (HOMO) or the lowest unoccupied molecular orbital (LUMO) of molecule. Within this model, which is a particular case of Fano resonance [9], we can calculate the elastic transmission function of the CNT. We have done this using the nonequilibrium Green's function (NEGF) formalism [10] as described in Refs. [11,12].

The calculated transmission function of the model [13] exhibits a dip in one of the two transport channels. The position of the dip is directly proportional to $\varepsilon_{0}$. For weak coupling, i.e., small values of $t$, the dip becomes more narrow and shallow and is located closer to $\varepsilon_{0}$, while for strong coupling the dip is wider with a depth approaching 1 (which means that one of the two transmission channels has been completely suppressed). In the strong coupling case the dip is located close to the on-site energy of the CNT sites which coincide with the Fermi level. 
We can understand this result and predict what happens when two molecules are chemisorbed, by rather simple means. Let us consider the subspace spanned by the four degenerate CNT Bloch states at the top of the valence band and the impurity state(s). The Hamiltonian matrix within this subspace is shown below in the case of a single impurity adsorbed at $\vec{R}=0$, and two impurities adsorbed at $\vec{R}=(0,0)$ (an $A$ site) and other at the general site $\vec{R}=$ $n \cdot \vec{a}_{1}+m \cdot \vec{a}_{2}$, respectively: (being $\vec{a}_{1,2}$ the basis vectors of graphene)

i) $\left(\begin{array}{ccccc}-\Delta & 0 & 0 & 0 & t \\ 0 & -\Delta & 0 & 0 & t \\ 0 & 0 & -\Delta & 0 & t \\ 0 & 0 & 0 & -\Delta & t \\ t & t & t & t & \varepsilon_{0}\end{array}\right)$

(ii) $\left(\begin{array}{cccccc}-\Delta & 0 & 0 & 0 & t & t \cdot f_{K_{+}} \\ 0 & -\Delta & 0 & 0 & t & t \cdot f_{K_{-}^{\prime}} \\ 0 & 0 & -\Delta & 0 & t & t \cdot f_{K_{+}^{\prime}} \\ 0 & 0 & 0 & -\Delta & t & t \cdot f_{K_{-}} \\ t & t & t & t & \varepsilon_{0} & 0 \\ t \cdot f_{K_{+}}^{*} & t \cdot f_{K_{-}^{\prime}}^{*} t \cdot f_{K_{+}^{\prime}}^{*} t \cdot f_{K_{-}}^{*} & 0 & \varepsilon_{0}\end{array}\right)$

where $f_{i}$ is the phase factor of the Bloch state at the position of the second impurity $\left(f_{i}\right.$ values are detailed in the section 2 of Ref. [13]). The parameter $2 \Delta$ represents the gap induced by the curvature in zigzag and chiral CNTs ( $\Delta=0$ for armchair CNTs) The eigensolutions of matrix (i) are shown in Table I. The eigenvectors 3, 4, and 5 represent nonpropagating states (they have equal weight on left- and right-moving Bloch states), while the eigenvectors 1 and 2 represent states propagating in the left and right directions, respectively. It is important to notice that the eigenvectors having zero weight on the impurity, in particular, states 1 and 2, are not only solutions in the restricted subspace but are in fact exact solutions to the full problem as they do not couple to the remaining Bloch states of the CNT. Consequently, state 1 (2) constitutes a channel for transporting electrons unhindered through the tube in the left (right) direction even in the presence of the impurity.

The solutions of the secular problem (ii) depend strongly on the position of the second impurity. We can distinguish three cases: (1) When the second impurity is at an $A$ site such that the relative position $\vec{R}$ fulfils the condition:

$$
\vec{R}=n \cdot \vec{a}_{1}+m \cdot \vec{a}_{2}, \quad n-m=3 p, \quad \forall p \in \mathbb{Z}
$$

all the $f_{i}$ parameters are 1 (for every kind of metallic CNTs) and the corresponding eigenvectors of (ii) have the same form as those of (i). Thus, in this case there are again two fully transmitting eigenstates (moving in the positive and negative directions of the tube, respectively) which can carry electrons unhindered through the CNT. The extra eigenvector as compared to (i) corresponds to the antibonding combination of the two impurity levels, without any weight from the CNT. It is instructive to notice that (2) is the same condition required by a CNT to be metallic $[14,15]$ (neglecting curvature/hybridization effects). (2) If the second impurity is placed at an $A$ site, but does not satisfy the condition (2) all the eigenvectors are nonpropagating (for every kind of metallic CNTs), and the transmission is completely suppressed. (3) If the second impurity is placed at a $B$ site the form of the eigenvectors depend strongly on the actual adsorption site. While there will be eigenstates with a nonzero momentum along the tube axis, none of these will be fully transmitting, i.e., have weight solely on either forward- or backward-moving Bloch states. Consequently the conductance is expected to fluctuate more or less randomly as the second adsorption site is varied over the $B$ sublattice.

More generally it can be shown that the propagating eigenstate $|\psi\rangle=-\frac{1}{\sqrt{2}}\left|K_{+}^{\prime}\right\rangle+\frac{1}{\sqrt{2}}\left|K_{+}\right\rangle$(eigenstate 2 of Table I) vanishes simultaneously at all the $A$ sites fulfilling the condition (2) and thus enables scattering-free transport through the CNT in the presence of an arbitrary number of impurities adsorbed at these special sites of the $A$ sublattice.

We mention that similar models, including the $\mathbf{k} \cdot \mathbf{p}$ equation and the Born approximation for pointlike scattering potentials, have previously been used to study the impurity scattering in a CNT [6].

In order to address the validity of the conclusions drawn from the simple TB model for scattering under more realistic conditions, we have performed first principles DFT + NEGF transport calculations following the approach described in Refs. [11,12] for a number of small molecules $\left(\mathrm{H}, \mathrm{COOH}, \mathrm{OH}, \mathrm{NH}_{2}\right.$ and $\mathrm{NO}_{2}$ ) chemisorbed on

TABLE I. Eigenvalues and (unnormalized) eigenvectors obtained after solving Eq. (1) (i). The eigenvectors are expressed as linear combinations of the four degenerate valence band eigenvectors at the graphene Brillouin Zone and the impurity level, $\left|\varepsilon_{0}\right\rangle . E^{ \pm}=$ $\underline{\varepsilon_{0}-\Delta \pm \sqrt{\varepsilon_{0}^{2}+\Delta^{2}+16 t^{2}+2 \Delta \varepsilon_{0}}}$.

\begin{tabular}{ccccccc}
\hline \hline & Eigenvalue & $\left|K_{+}\right\rangle$ & $\left|K_{-}^{\prime}\right\rangle$ & $\left|K_{+}^{\prime}\right\rangle$ & $\left|K_{-}\right\rangle$ & $\left|\varepsilon_{0}\right\rangle$ \\
\hline$(1)$ & $-\Delta$ & 0 & 1 & 0 & -1 & 0 \\
$(2)$ & $-\Delta$ & -1 & 0 & 1 & 0 & 0 \\
$(3)$ & $-\Delta$ & -1 & 1 & -1 & 1 & 0 \\
$(4)$ & $E^{+}$ & $t /\left(E^{+}+\Delta\right)$ & $t /\left(E^{+}+\Delta\right)$ & $t /\left(E^{+}+\Delta\right)$ & $t /\left(E^{+}+\Delta\right)$ & 1 \\
$(5)$ & $E^{-}$ & $t /\left(E^{-}+\Delta\right)$ & $t /\left(E^{-}+\Delta\right)$ & $t /\left(E^{-}+\Delta\right)$ & $t /\left(E^{-}+\Delta\right)$ & 1 \\
\hline \hline
\end{tabular}


the $(6,6)$ armchair, $(9,0)$ zigzag, and $(8,2)$ chiral CNTs [13]. We have chosen these CNTs due to their small radii which make them more reactive towards binding molecules at the sidewall as compared to large radius CNTs [16] (curvature induces small $s p^{3}$-like hybridization).

All molecules bind covalently to an on-top site of the CNT wall. The calculated distances between the adsorbates and the CNT, $d_{i}$, are (in $\AA$ ) $d_{\mathrm{H}}=1.18, d_{\mathrm{COOH}}=1.57$, $d_{\mathrm{OH}}=1.45, d_{\mathrm{NO}_{2}}=1.64$ and $d_{\mathrm{NH}_{2}}=1.49$. We have also calculated the distances and transmission curves for $\mathrm{CO}$ and $\mathrm{CO}_{2}$. These molecules are physisorbed by the $\mathrm{CNT}$ at $d_{\mathrm{CO}}=2.55$ and $d_{\mathrm{CO}_{2}}=2.52$, and we have found that they do not influence the transport properties of the CNT close to the Fermi level [13]. For this reason we focus on the chemisorbed species in the following.

In Fig. 1 we show the effect of a single chemisorbed molecule on the transmission function for the different CNTs. For all molecules the effect is to reduce the transmission function from $2 G_{0}$ to $1 G_{0}$ in the vicinity of the Fermi level, in agreement with the TB results for the strong coupling case (see Fig. 2 in the supplementary material [13]). The same effect was observed by Lee et al. for the phenyl molecule adsorbed on top of a carbon atom in a $(5,5)$ CNT [16]. This happens because one of the two channels which are available in the pure CNT around the Fermi level is almost completely suppressed by scattering off the adsorbate while the other remains unaffected. This is clearly seen by decomposing the transmission function into the nonmixing eigenchannels [17]. The contributions from individual eigenchannels to the total transmission are shown in the Top-Right Fig. 1. In Figs. 2(a) and 2(b) we show the contour surfaces of the fully transmitting and

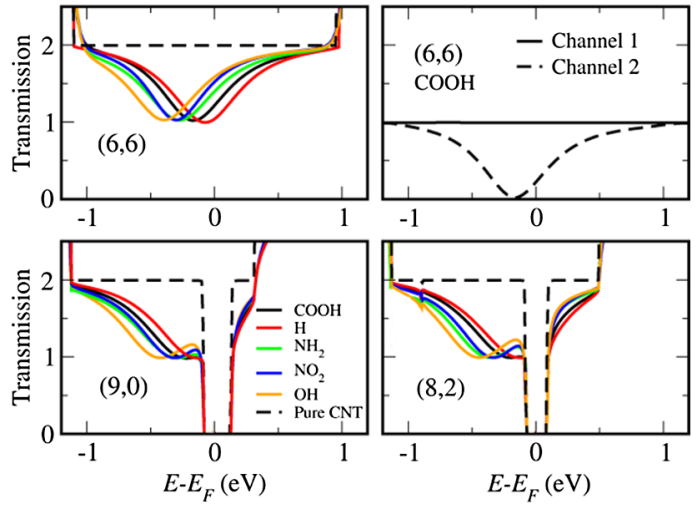

FIG. 1 (color online). Top-Left: Transmission curves for a $(6,6)$ carbon single wall nanotube (SWNT) with a single molecule chemisorbed on top of a $\mathrm{C}$ atom: $\mathrm{COOH}$ (black), $\mathrm{H}$ (red), $\mathrm{NH}_{2}$ (green), $\mathrm{NO}_{2}$ (blue) and $\mathrm{OH}$ (orange). The transmission curve for the pure nanotube is also shown (black dashed line). Bottom-Left: The same for a $(9,0)$ CNT. Bottom-Right: The same for a $(8,2)$ CNT. Top-Right: Transmission of the different eigenchannels for a $(6,6) \mathrm{CNT}$ with a single $\mathrm{COOH}$ molecule adsorbed. Notice that channel 1 (solid line) is completely unaffected by the impurity, while channel 2 (dashed line) is completely suppressed in the vicinity of the Fermi level. blocked eigenchannels at the Fermi energy. Notice that the fully transmitting channel vanishes at $A$ sites fulfilling the condition (2) as predicted by the TB model.

It is interesting to notice that, despite the fact that the molecules bind to the CNT via an $\mathrm{H}, \mathrm{C}, \mathrm{O}$, and $\mathrm{N}$ atom, respectively, the effect of the adsorbates on the transmission is very similar, the only difference being a slight shift in the position of the dip. Clearly, these results are in line with the predictions of the TB model for the strong coupling regime [13].

We next consider what happens when two molecules are adsorbed at different sites of the CNT. We adsorb the first molecule at an $A$ site, and vary the adsorption site for the second molecule. We distinguish between the cases where both molecules are located on $A$ sites and cases where the second molecule is adsorbed at a $B$ site. We have performed calculations for various adsorption sites for all molecular species as well as for combinations of those molecules. As in the case of a single molecule, the shape of the transmission function is insensitive to the adsorbed species. Again this indicates that the transport properties of metallic CNTs are affected in a similar way by different chemisorbed molecules. Having observed this, we focus to the simplest case of adsorbed $\mathrm{H}$ in what follows. The results for $\mathrm{H}$ adsorbed at different $A-A$ configurations on the armchair, zigzag, and chiral CNTs are shown in Fig. 3. It is easy to distinguish two different types of transmission curves: For the cases fulfilling Eq. (2) the transmission at the Fermi level is close to 1 [18]. For the cases not fulfilling Eq. (2) the transmission at the Fermi level almost completely suppressed (less than $0.1 G_{0}$ ) [19].
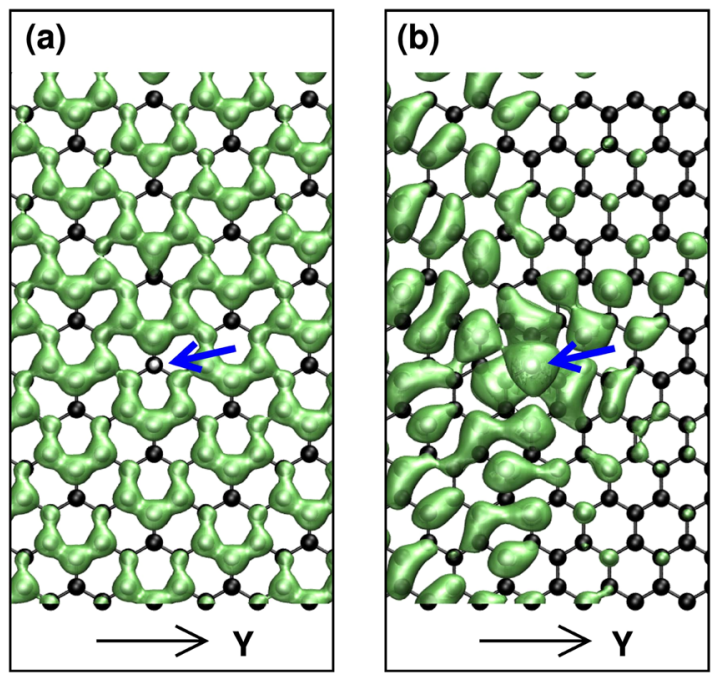

FIG. 2 (color online). Contour surfaces of the two eigenchannels at the Fermi level of a $(6,6)$ CNT with a single hydrogen atom adsorbed on top of a $\mathrm{C}$ atom. The states correspond to channels 1 and 2 in the top-right panel of Fig. 1. The position of the hydrogen atom is indicated by the blue arrow. The CNT is shown unrolled for clarity. (a) Fully transmitting eigenchannel, $T=1$. (b) Blocked eigenchannel, $T=0$. Notice that channel 1 has zero weight on all sites fulfilling Eq. (2). 


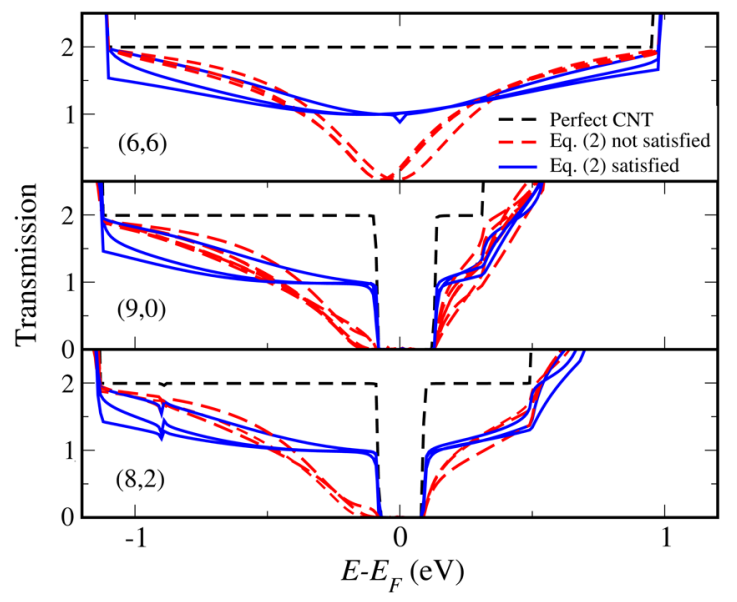

FIG. 3 (color online). Top: Transmission functions for a $(6,6)$ carbon SWNT with two hydrogen atoms adsorbed at different $A$ sites. The six inequivalent $A-A$ configurations in one unit cell of the CNT are shown. The blue solid lines correspond to the cases where Eq. (2) is fulfilled. The red dashed lines correspond to the cases where Eq. (2) is not fulfilled. The transmission function for the pure CNT is also shown (black dashed line) Middle: The same for a $(9,0) \mathrm{CNT}$. We show the 9 inequivalent $A-A$ configurations in one unit cell of the CNT. Bottom: The same for a $(8,2)$ CNT. We show out 6 of the 14 inequivalent $A-$ $A$ configurations within a CNT unit cell.

In contrast there is no trend in the transmission functions of the $A-B$ configurations [13], and the transmission values at the Fermi level fluctuate randomly between 0 and $2 G_{0}$. Similar fluctuations were observed by Lee et al., who considered only $A-B$ configurations of phenyl pairs [16].

We have made several tests to study the robustness and universality of the observed effects. When two different molecular species are adsorbed, the transmission dips become slightly wider in all cases. In some of the cases where Eq. (2) is not fulfilled, the transmission is not always fully blocked, but has a minimum of $0.25 G_{0}$ rather than $0.1 G_{0}$. This is due to the different position of the HOMO levels of the two molecules. We also analyzed the effect of temperature by making random displacements of the atoms corresponding to $300 \mathrm{~K}$ and found only minor changes in the transmission functions. However, replacing the adsorbates by vacancies produces more long ranged perturbations and destroys the effect described here. Although the present calculations are based on an independent particle approximation, electron-electron interactions are not expected to affect the general trends discussed here which mainly results from the symmetry of the lattice and the wave functions (see Ref. [20] and references therein for a discussion of many-body effects in quantum transport in nanostructures).

There are two main conclusions of this Letter. First, when a single molecule is chemisorbed on the sidewall of a metallic CNT, one of its two transmission channels close to the Fermi level is blocked while the other remains unaffected. We have found that this behavior is quite universal in the sense that the depth and position of the transmission minimum depends only weakly on the molecular species. Second, it is possible to adsorb an arbitrary number of molecules on the CNT sidewall and still conserve a fully open transport channel. To obtain this, all molecules must be adsorbed on the same sublattice and their relative positions should fulfill Eq. (2). On the other hand, if a single molecule is adsorbed outside this array of special sites, both channels are almost completely blocked and the conductance drops sharply, independently of tube chirality.

[1] J. C. Charlier, X. Blase, and S. Roche, Rev. Mod. Phys. 79, 677 (2007).

[2] K. Balasubramanian and M. Burghard, Small 1, 180 (2005).

[3] C. Hierold, Carbon Nanotube Devices: Properties, Modeling, Integration and Applications (Wiley-VCH, Weinheim, 2008).

[4] P. G. Collins et al., Science 287, 1801 (2000).

[5] H. J. Choi et al., Phys. Rev. Lett. 84, 2917 (2000).

[6] T. Ando and H. Suzuura, J. Phys. Soc. Jpn. 71, 2753 (2002); K. Akimoto and T. Ando, J. Phys. Soc. Jpn. 73, 2194 (2004); Q. Zhang et al., Phys. Rev. B 73, 235438 (2006).

[7] A. Rochefort et al., Phys. Rev. B 60, 13824 (1999).

[8] R. Tamura, Y. Sawai, and J. Haruyama, Phys. Rev. B 72, 119903 (2005).

[9] U. Fano, Phys. Rev. 124, 1866 (1961).

[10] Y. Meir and N.S. Wingreen, Phys. Rev. Lett. 68, 2512 (1992).

[11] K. S. Thygesen and K. W. Jacobsen, Chem. Phys. 319, 111 (2005).

[12] M. Strange et al., J. Chem. Phys. 128, 114714 (2008).

[13] See EPAPS Document No. E-PRLTAO-101-046846 for auxiliary figures. For more information on EPAPS see (http://www.aip.org/pubservs/epaps.html).

[14] N. Hamada, S. Sawada, and A. Oshiyama, Phys. Rev. Lett. 68, 1579 (1992).

[15] J. W. Mintmire, B. I. Dunlap, and C. T. White, Phys. Rev. Lett. 68, 631 (1992).

[16] Y. S. Lee, M. B. Nardelli, and N. Marzari, Phys. Rev. Lett. 95, 076804 (2005).

[17] M. Brandbyge, M. R. Sorensen, and K. W. Jacobsen, Phys. Rev. B 56, 14956 (1997).

[18] In comparison with the case of one single molecule adsorbed (Fig. 1) the width of the dip is broadened, due to the fact that the suppressed channel is scattered by two molecules. However, as in Fig. 1, one channel remains completely open.

[19] We have checked these properties when the two molecules are in different unit cells and also for the case with three molecules in the same sublattice. Again one channel remains open if condition 2 is satisfied and both channels are blocked if it is not.

[20] K. S. Thygesen and A. Rubio, Phys. Rev. B 77, 115333 (2008). 